\title{
Prevention of Renal Stone Recurrence: Knowledge and Practice Patterns Among Medical Officers of DHQ Hospital, Faisalabad
}

\section{Muhammad Saifullah ${ }^{1}$, Hanan Noor ${ }^{2}$, Muhammad Waqas Iqbal ${ }^{3}$, Nauman Khalid ${ }^{4}$, Muhammad Sohail $^{5}$, Muhammad Akmal ${ }^{6}$, Ghulam Mehboob Subhani ${ }^{7}$}

\begin{abstract}
Objective: To assess the knowledge and practice patterns among medical officers of DHQ Hospital, Faisalabad in the prevention of recurrent renal stones. Cross sectional study. DHQ hospital, Faisalabad during 01-01-2019 to 30-09-2019.

Methods: In this study the medical officers of either gender and age more than 25 years and working in DHQ Hospital, Faisalabad were included. They were assessed on the basis of a designed questionnaire regarding their general practice and knowledge in order to prevent renal stone recurrence. The paper based questionnaire was circulated to medical officers in different departments of the hospital.

Results: In this study total 225 medical officers that were included. $135(60 \%)$ of these participants were males and $90(40 \%)$ were females. The mean age of the participants was $29.70 \pm 3.21$ years. The mean working experience was $4.40 \pm 2.18$ years. On assessment, $65.67 \%$ medical officers had adequate knowledge regarding recurrent renal stone prevention according to latest practice guidelines. Unfortunately $62.22 \%$ respondents were not practicing their knowledge adequately.

Conclusion: The knowledge regarding the clinical practices in light of recent guidelines was optimal, however the practice regarding the preventive strategies was below power.

Keywords; Renal stone, Recurrence, Medical officer, prevention.
\end{abstract}

\section{Introduction}

Tephrolithiasis is an ancient disease which is 1 known to humans for centuries. It affects 5-20\% of mankind at least once during their life. ${ }^{1,2}$ It is a widespread disease affecting young males more than females. ${ }^{3,4} 12 \%$ of males and $7 \%$ of females develop renal stone disease during their life. ${ }^{5}$ It is one of the commonest urological diseases with its incidence increasing all across the globe. It has been considered

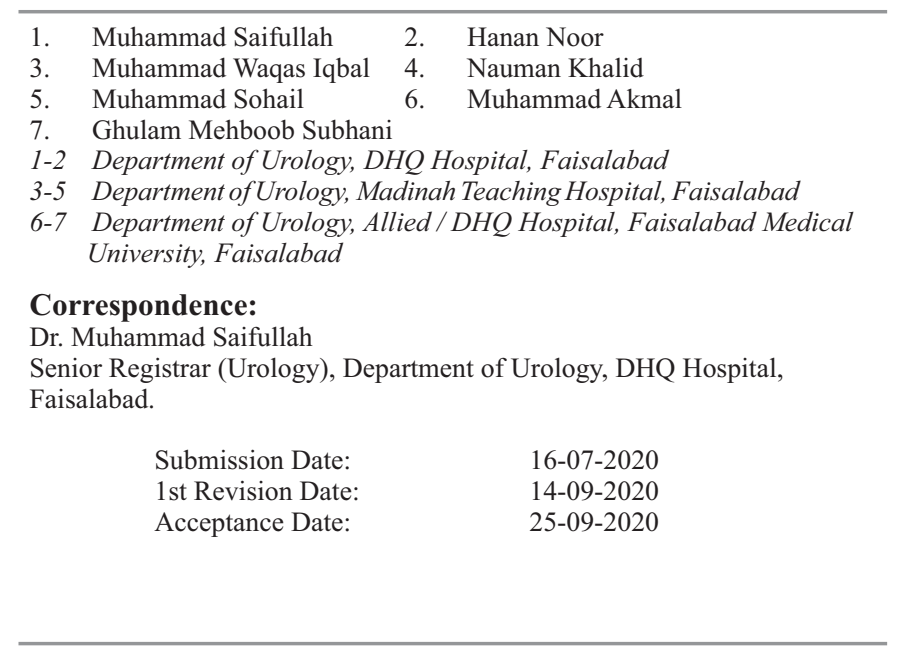

a lifelong disease as it has higher chances of recurrence with poor clinical consequences. ${ }^{6}$ These consequences include chronic renal failure, end-stage kidney disease, diabetes mellitus, hypertension and cardiovascular accidents. ${ }^{1}$

Stone formation is usually asymptomatic initially. However, with the passage of time as the stone size progresses, it may cause colicky flank pain, hematuria, urinary tract infection and hydroureteronephrosis ${ }^{1}$. Calcium based stones are the most prevelant type of stones accounting for $70 \%-85 \%$ of cases. $^{3,7,8}$ Non-calcium stones include urate stones (5\%-10\%), magnesium ammonium phosphate stones $(1 \%-5 \%)$, cysteine stones $(1 \%)$ etc. ${ }^{3}$ Stone analysis is valuable tool used for diagnosing underlying etiological risk factors which lead to renal stone formation, especially in rare stone varieties like urate, ammonium urate, cysteine, drug-induced and infectioninduced stones. ${ }^{5}$ Different methods used for stone analysis include infrared spectroscopy, X-ray diffraction and polarizing microscopy. 
Lifetime recurrence rate in first time stone formers is $50 \%$. Time between recurrence episodes decreases with every passing episode. ${ }^{2}$ Metabolic workup is compulsory for recurrent stone formers $\&$ in special circumstances for first time stone formers. ${ }^{7}$ According to one study, advice of 24-hour urinary metabolic workup was followed only by $7 \%$ of symptomatic patients and $17 \%$ of patients with recurrent urolithiasis for prevention purpose.'

The significance of adopting preventive measures can be assessed by the recurrence rate of this disease. Effective utilization of the preventive strategies have reduced 5-year recurrence rate by $60 \%{ }^{2}$ However, prevention of recurrent stone disease is always a dilemma for patients as well as health care providers. It is not possible to prevent stone recurrence without knowing the pathophysiology which leads to stone formation ${ }^{1}$. Sedentary life style and modern eating habits like fast foods, carbonated drinks, animal proteins, dairy products, inadequate water intake, high sodium diet, less vegetable consumption etc contribute in stone formation. ${ }^{4,8}$

To address this issue, we carried out a survey to evaluate the knowledge, attitudes and practice patterns among medical officers of DHQ hospital, Faisalabad regarding prevention of renal stone recurrence. The rationale of our study was to find out the knowledge of medical officers working in our hospital regarding prevention of renal stone recurrence. Furthermore, we needed to assess their attitude towards the recent guidelines and application of their knowledge in every day practice.

\section{Objective}

To assess the knowledge and practice patterns among medical officers of DHQ Hospital, Faisalabad in the prevention of renal stone recurrence.

\section{Methods}

This was a cross sectional study that was conducted at DHQ Hospital, Faisalabad during 01-01-2019 to 3009-2019. In this study, 225 medical officers of either gender or age more than 25 years and working in DHQ Hospital, Faisalabad were included using consecutive non-probability sampling technique. After taking informed consent, medical officers were assessed on the basis of a designed questionnaire regarding their general practice and knowledge about prevention of renal stone recurrence. The questionnaire was designed according to the latest guidelines of European Association of Urology and World Health Organization regarding recurrent renal stone prevention. This included questions regarding 2 domains: knowledge and practice patterns. Demographic profile was completed. Questions regarding practice were assessed before the knowledge assessment, so that respondents may not take any leading answers. Practice and knowledge was assessed using multiple-choice questions. The paper based questionnaire was circulated to medical officers working in different departments of the hospital. SPSS-version 23.0 was used for data analysis and frequency and percentages were calculated for categorical data and mean and standard deviation for quantitative variables.

\section{Results}

In this study total 225 medical officers were included. There were 135 (60\%) males and 90 (40\%) females (Figure \# 1). The mean age of the participants was $29.70 \pm 3.21$ years. The mean working experience was $4.40 \pm 2.18$ years with a range of 2 to 9 years as shown in table \# 1 .

Regarding knowledge, $55.56 \%$ of medical officers stated that high urine output is the most important inhibitory factor for stone formation (Figure \# 2). we further noted that $68.89 \%$ medical officers were aware of the fact that high fluid intake is the single most important dietry modification which prevents recurrence of urinary stone disease (Figure \# 3). $56.17 \%$ medical officers correctly identified the comorbid factors which lead to urinary stone formation. Salt intake reduction was advocated by only $39.57 \%$ $(n=93)$ medical officers. $54.44 \%$ respondents $(n=$ 128) were not aware about protein restriction in diet of stone formers (Figure \#4). 78.89\% $(n=177)$ clinicians could not correctly identify the commonest recurring stones i.e., uric acid stones.

Table 1: Demographics of Study Subjects

\begin{tabular}{lcc}
\hline \multicolumn{1}{c}{ Variables } & Mean \pm SD & Range \\
\hline Age & $29.70 \pm 3.21$ & $25-34$ \\
Working Experience & $4.40 \pm 2.18$ & $2-9$ \\
\hline
\end{tabular}

$26.38 \% \quad(n=62)$ medical officers advised stone analysis to every first time stone former who mana- 
ged to retrieve the stone. Animal protein restriction was advised by only $24.44 \%(n=55)$ medical officers. Maintenance of normal calcium requirements was practiced by $45.33 \% \quad(\mathrm{n}=102)$ medical officers. Overall, 65.67\% ( $\mathrm{n}=154)$ medical officers had adequate knowledge regarding recurrent renal stone prevention according to latest practice guidelines. Unfortunately $62.22 \%(n=146)$ respondents were not practicing their knowledge adequately (Figure \# 5). The value of pearson correlation coefficient $(R)$ is 0.4262 . By this we conclude that there is a weak positive correlation between knowledge \& practice. The $\mathrm{p}$-value is $<0.00001$ which is significant.

Figure \# 1: Distribution of participants according to Gender
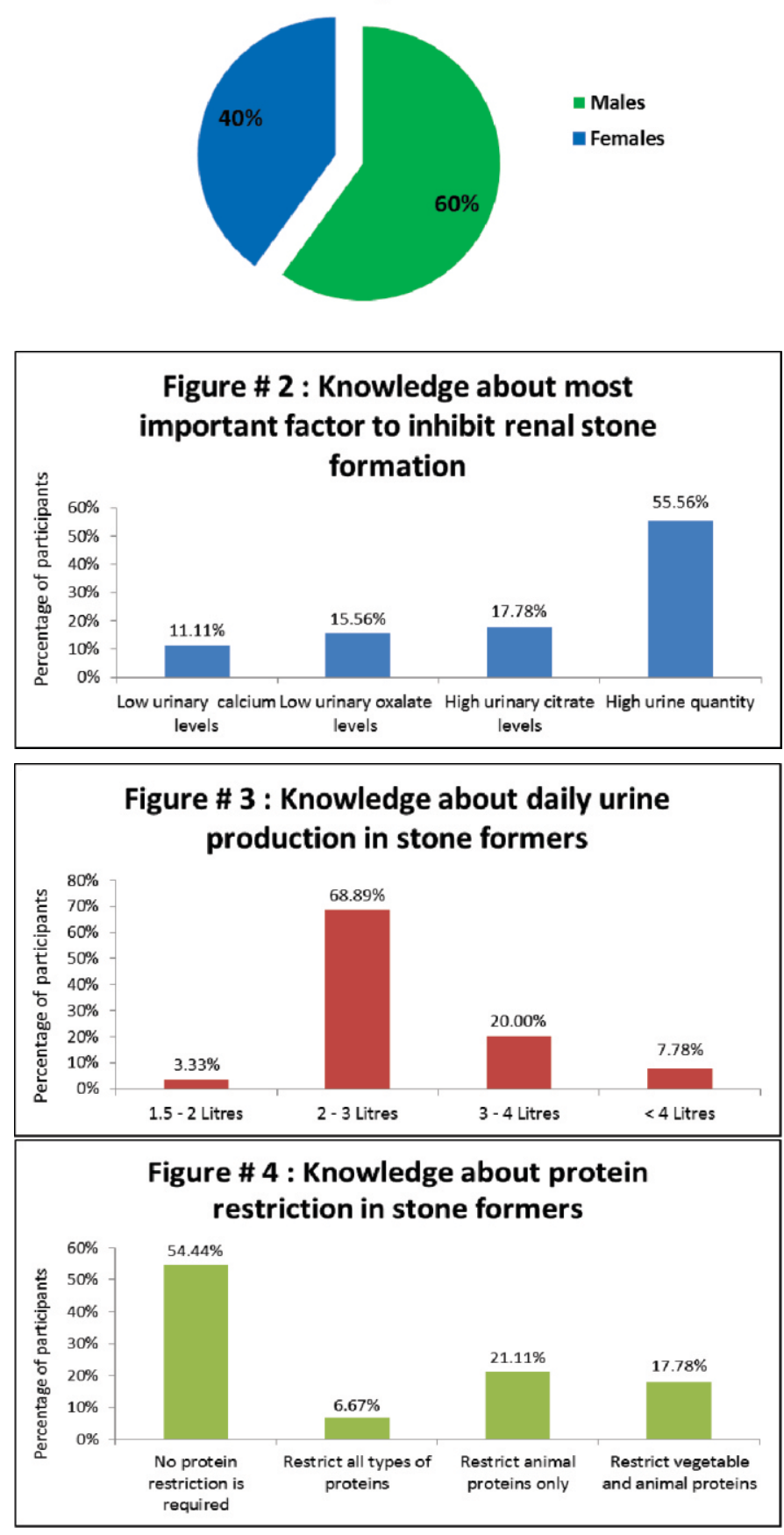

\section{Discussion}

Knowledge, Attitude and Practices (KAP) survey is a method utilized for assessment of different diseases by using predefined standardized questionnaires. There are only a few studies which address this issue. Respondents of our study did not clinically practice the preventive strategies regarding recurrent renal stones despite having adequate knowledge. Although $65.67 \%$ respondents had satisfactory knowledge regarding prevention of recurrent nephrolithiasis, only $37.78 \%$ medical officers used their knowledge productively in practice. Lack of utilization of knowledge is attributable to factors like disagreement of clinicians with guidelines and lesser outcomes than expectations.

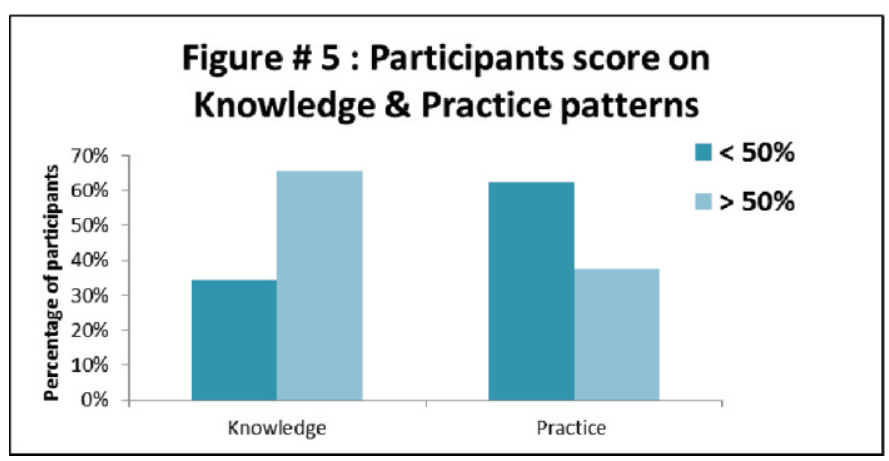

Different studies have documented that recurrence of kidney stone disease is remarkably lower in patients who ingest high levels of fluids. ${ }^{10,11}$ Therefore, increasing fluid consumption is the most important recommendation in prevention of urolithiasis. Current guidelines suggest maintenance of 2 to $3 \mathrm{~L}$ of urine volume per day. Stone formers have a very low urine output which is $1.6 \mathrm{~L} /$ day on average. ${ }^{12,13}$ Decreased urinary output is due to decreased fluid intake which leads to increased concentration of solutes in urine which crystallize to form stones. ${ }^{13}$ In our study, urine output of 2 to 3 litres per day which is required for prevention of urolithiasis was supported by $68.89 \%$ participants. However, Bos D et al noted that only $20 \%$ of participants advocated $2-3$ litres per day of urine output. Generally, adequate fluid intake of 2.5-3 liters a day, intake of moderate amount of animal protein, dietary calcium, vitamins/mineral supplements, fruits/vegetables and weight loss are safe and reasonable dietary recommendations as per guidelines. ${ }^{2}$

Urate stones accounts $5-10 \%$ of all renal stones and $10-15 \%$ of all urinary stones. ${ }^{11,14}$ High body mass index, much intake of fructose rich products, and high 
protein intake has a significant association with the formation of uric acid stones. ${ }^{11}$ Higher incidence of urate stones is commonly associated with increased urinary uric acid levels, persistent low $\mathrm{pH}$ of urine and decreased urine volume. Uric acid stones are notorious for their recurrence. Obesity, diabetes, environmental factors \& change in dietry habits also increase risk of stone formation significantly. ${ }^{14}$ Bos D et al concluded that more than $90 \%$ respondents were unaware of the recurrent nature of uric acid stones. ${ }^{2}$ However, we studied that $78.89 \%$ clinicians could not identify uric acid stones as the commonest recurrent stones.

Oxalate plays an important role in formation of calcium stones. Foods rich in oxalates includes rhubarb, beets, nuts, cranberries, chocolates, wheat bran, spinach, sesame seeds, tomatoes, tea and green beans increases the risk of kidney stones. ${ }^{13,15}$ Critical factor in formation of calcium oxalate stone is the amount of oxalate excreted in urine. ${ }^{16}$ The concentration of calcium is considerably higher than the concentration of oxalate in urine. Therefore, change in concentration of oxalates have a more impact than change in concentration of calcium on the supersaturation of crystals of calcium oxalate. For attenuating calcium oxalate supersaturation one should take normal amount of dietry calcium and reduce the intake of high-oxalate-containing foods, thus reducing stone progression in kidney stone-forming individuals. ${ }^{16}$ Current guidelines suggest oxalate-rich foods in moderate amount and calcium intake adjusted to 1000-1200 mg/day. ${ }^{16}$ In stone prevention, limiting intake of sodium in diet is a key factor. ${ }^{17}$ Recurrent calcium nephrolithiasis can be reduced by reduction in dietry sodium intake i.e., 1.8-2.3 g/day. According to the European Association of Urology guidelines, the daily intake of salt $(\mathrm{NaCl})$ should limit to 4-5 grams. Volume expansion due to high sodium intake decreases sodium and calcium reabsorption in proximal tubules and thus enhances calcium excretion rendering urine more lithogenic. ${ }^{12,13}$ Increased excretion of sodium in urine leads to increased saturation of monosodium urate. This acts as a nidus for calcium crystal formation. In our study, salt intake reduction was advised by only $39.57 \%$ ( $\mathrm{n}=93$ ) medical officers.

Cranberry juice has been reported to significantly alter key risk factors for urolithiasis as it increases urinary citrate excretion which inhibits calcium litho- genesis and decreases the excretion of phosphate and oxalates in urine. Furthermore, it has been reported that there is reduction in saturation of calcium oxalate with the use of cranberry juice. ${ }^{18}$ Diets rich in animal proteins should be restricted in kidney stones patients. High amount of calcium and urate is excreted in urine by consuming diet rich in animal proteins from meat, fish and chicken. Purine rich products like red meat, tinned fish, meat extracts and muscles also increases the uric acid concentration, therefore intake of these products should be restricted to $1 \mathrm{gm} / \mathrm{kg} /$ day. ${ }^{13}$ An acid load is generated by consumption of animal proteins which increases excretion of urinary calcium and reduces the excretion of calcium stone inhibitor, citrate. However, in our study only $24.44 \%$ $(n=55)$ medical officers correctly identified that animal protein restriction is mandatory for recurrent stone formers.

All renal stone patients should undergo metabolic analysis to rule out systemic disease as advocated by CUA. Stone-formers should undergo metabolic evaluation which include serum analysis (sodium, chloride, potassium, calcium, bicarbonate, albumin, creatinine, uric acid and phosphate), a urinalysis and a stone analysis. Bos D et al noted that $57 \%$ health care workers quoted multiple stones as an indication for stone analysis. ${ }^{2}$ In our study, only $26.38 \%$ medical officers ordered stone analysis to first time stone formers.

Diet plays a very important role in urinary calcium containing stone formation. Calcium oxalate stones account for $75 \%$ of calcium containing urinary stones. ${ }^{18}$ Diet low in calcium is discouraged as it allows more absoption of oxalates which leads to hyperoxaluria. Therefore, moderate calcium in diet ( $1 \mathrm{~g} /$ day) is usually recommended for patients. Intake of supplemental calcium may increase the risk of symptomatic kidney stone while dietry calcium intake decreases the risk. Maintenance of normal calcium intake (1-1.2g/day) was practiced by $45.33 \%$ $(n=102)$ medical officers in our study.

In this study, we noted that medical officers did not practice latest guidelines despite having adequate knowledge. Improper utilization of preventive measures is attributable to different factors which include lack of awareness, disagreement of clinicians with guidelines, lesser outcomes than expectations and 
absent incentives which can improve delivery of preventive system.

In one study, it is declared knowledge of urologists in Saudi Arabia as deficient. They recommended activities to improve their knowledge. They further proposed that application of knowledge in routine practice should be enforced. ${ }^{17}$ In another study it was noted that patients lack adequate knowledge about the dietry modifications required by the recurrent stone formers. Even educated people lacked sufficient information. ${ }^{19}$ It was recommended in a similar study that planned educational activities offered on regular basis to the patients to improve the self care practices and knowledge. ${ }^{20}$ Moreover, it was concluded through another study that patients having history of stone disease were more knowledgeable and careful in preventing recurrent stone formation. He advised interventions for improving preventive measures as well as correcting misconceptions. ${ }^{21}$

As described, many international studies have elaborated the important of preventive strategies in recurrent urolithiasis. But we have studied those healthcare professionals who are usually the first respondents to the patients. They have adequate knowledge regarding prevention of urinary stone disease. However, despite of adequate knowledge, the pattern of their clinical practices are not according to the latest guidelines.

\section{Conclusion}

The knowledge regarding the clinical practices in light of recent guidelines was optimal, however the practice regarding preventive strategies was suboptimal.

\section{Recommendations}

We recommend more frequent awareness programs for prevention of renal stone recurrence in order to improve the knowledge of the healthcare providers. This will in turn encourage young medical officers to incorporate their knowledge in daily practice regarding recurrence of renal stone preventive strategies like dietry modifications and fluid intake etc

\section{Conflict of Interest}

There are no conflict of interest.
Author's Contribution:

MS: Principal Author \& Manuscript Writer

HN: Investigator, Data Collector

MWI: Literature Review and Data Analysis

NK: Statistical Analysis

MS: Proof Reading

MA, GMS: Research Supervision

\section{References}

1. Alelign T, Petros B. Kidney Stone Disease: An Update on Current Concepts. Advances in Urology. 2018;2018:1-12.

2. Bos D, Abara E, Parmar M. Knowledge, attitudes, and practice patterns among healthcare providers in the prevention of recurrent kidney stones in Northern Ontario. Canadian Urological Association Journal. 2014;8:795-804.

3. Sorokin I, Pearle M. Medical therapy for nephrolithiasis: State of the art. Asian Journal of Urology. 2018;5:243-55.

4. Moussa M, Abou Chakra M. Patient's perception of kidney stone prevention within the emergency department and its adherence factors: a single institution study. BMC Emergency Medicine. 2019;19(1).

5. Spivacow FR, Valle EEDEL, Lores E, Rey PG. Kidney stones: composition, frequency and relation to metabolic diagnosis. Med (Buenos Aires). 2016; 76:343-8.

6. Zisman AL. Effectiveness of Treatment Modalities on Kidney Stone Recurrence. Clin J Am Soc Nephrol. 2017;12:1699-708.

7. Zeng J, Wang S, Zhong L, et al. A Retrospective Study of Kidney Stone Recurrence in Adults. J Clin Med Res. 2019;11:208-12.

8. Assadi F, Moghtaderi M. Preventive Kidney Stones: Continue Medical Education. Int J Prev Med. 2017; $8: 67$.

9. Gul Z, Monga M. Medical and dietary therapy for kidney stone prevention. Korean J Urol. 2014;55: 775-9.

10. Cheungpasitporn W, Rossetti S, Friend K, Erickson SB, Lieske JC. Treatment effect, adherence, and safety of high fluid intake for the prevention of incident and recurrent kidney stones: a systematic review and meta-analysis. J Nephrol. 2016;29:211-9.

11. Davis L. Nutrition Management of Uric Acid Stones. Pocket Guide to Kidney Stone Prevention. 2014;:7580.

12. Aggarwal R, Srivastava A, Jain SK, Sud R, Singh R. Renal stones: a clinical review. EMJ Urol. 2017;5:98- 
103.

13. Sofia NH, Walter TM. Prevalence and risk factors of kidney stone. Global J Res Analysis. 2016;5:183-7.

14. Ma Q, Fang L, Su R, Ma L, Xie G, Cheng Y. Uric acid stones, clinical manifestations and therapeutic considerations. Postgraduate Medical Journal. 2018;94: 458-62.

15. Holmes R, Knight J, Assimos D. Lowering urinary oxalate excretion to decrease calcium oxalate stone disease. Urolithiasis. 2015;44:27-32.

16. Mitchell T,Kumar P,Reddy T, Wood KD,Knight J, Assimos DG, Holmes RP. Dietary oxalate and kidney stone formation. Am J Physiol Renal Physiol. 2019; 316:409-13.

17. Binsaleh S, Habous M, Madbouly K. Knowledge, attitudes, and practice patterns of recurrent urinary stones prevention in Saudi Arabia. Urolithiasis, 2016;44:135-43.
18. Abbas W, Akram M, Sharif A. Nephrolithiasis; prevalence, risk factors and therapeutic strategies: a review. Madridge J Intern Emerg Med. 2019;3:90-5.

19. Alghamdi SYS, Alamri AM, Alzahrani RA, Alghamdi AH, Alghamdi AA, Alghamdi AA, et al. Awareness about symptoms and role of diet in renal stones among the general population of Albaha city. The Egyptian Journalof Hospital Medicine 2018; 70 : 50-59.

20. Mahmoud MH, Ramadan EN, Taha AS. Effectiveness of self-care intervention for patients with urolithiasis on their practices regarding nutrition. American J Nurs Res. 2019;7:856-69.

21. Almuhanna AM, Alomar M, Alsalman HK, AlMutayliq AA, Alnasser KA. Public awareness towards renal stone causes, symptoms and management amongst Saudis. Egyptian J Hosp Med. 2018; 70: $544-8$. 